\title{
Discard of organs is the Achilles heel of deceased donor organ transplantation program: a study of ethology and predictors of organ discard from South India
}

Banigallapati Vijay Kiran

Department of Nephrology, Nizam's Institute of Medical Sciences, Hyderabad, India

Background: With the significant growth of the deceased donor organ transplantation programs world-wide, the discard of organs also increased. Our objective was to assess the etiology and predictors of organ discard in Jeevandan-the deceased donor organ transplantation program from South India.

Methods: This was a retrospective chart analysis. Univariate and multivariate logistic regression analysis was used to predict the indicators of organ discard.

Results: The kidney discard rate and liver discard rate was $19.27 \%$ and $7.68 \%$, respectively. The main reason for kidney discard was marginal donor kidney (61.23\%) followed by chronic kidney disease in the donor (15.94\%). For liver discard, the main reason was cirrhosis of the liver (34.54\%) followed by non-alcoholic fatty liver disease (20\%). The risk factors for kidney discards were higher age, male gender, intracerebral hemorrhage as a cause of brain death, long-standing diabetes, hypertension, and elevated terminal creatinine. Protective factors against kidney discard were higher serum proteins and higher sodium. Risk factors for liver discards were history of alcohol abuse, higher bilirubin, higher alanine aminotransferase, and acute kidney injury. Higher platelet count is a novel protective factor identified.

Conclusions: We assessed the etiology for discard of kidneys and livers in a deceased donor organ transplant program. The factors that predict the discard and protective factors against discard were identified. 